\title{
Point-Contact Transport Properties of Strongly Correlated Electrons on Liquid Helium
}

\author{
D. G. Rees, ${ }^{1, *}$ I. Kuroda, ${ }^{1,2}$ C. A. Marrache-Kikuchi, ${ }^{1, \dagger}$ M. Höfer, ${ }^{3}$ P. Leiderer, ${ }^{3}$ and K. Kono ${ }^{1,2}$ \\ ${ }^{1}$ Low Temperature Physics Laboratory, RIKEN, Wako 351 0198, Japan \\ ${ }^{2}$ Department of Physics, Tokyo Institute of Technology, Tokyo 152 8551, Japan \\ ${ }^{3}$ Faculty of Physics, University of Konstanz, 78457 Konstanz, Germany
}

(Received 14 September 2010; published 14 January 2011)

\begin{abstract}
We present transport measurements of a nondegenerate two dimensional electron system on the surface of liquid helium at a point constriction. The constriction is formed in a microchannel by a split gate beneath the helium surface. The electrostatic energy of the electron system, which depends in part on the electron density, determines the split gate voltage threshold of current flow through the constriction. Steplike increases in conductance are observed as the confinement strength is reduced. As the Coulomb interaction between electrons is strong, we attribute this effect to the increase in the number of electrons that can pass simultaneously through the constriction. Close to the threshold, single electron transport is observed.
\end{abstract}

DOI: 10.1103/PhysRevLett.106.026803

PACS numbers: 73.20. r, 73.23. b, 85.30.Hi, 45.50.Jf

Either the quantum or classical transport of particles through constrictions is of fundamental importance in many physical systems. Classical dynamics in confined geometries have been studied in vortex matter in superconducting films [1] and in colloidal systems confined to quasi-1D channels [2]. Coulomb systems at constrictions have been studied using molecular dynamics simulations [3]. As long-range interactions are common in nature, these dynamics may have implications in a wide variety of systems such as the transport of ions through cell membranes [4]. Pedestrians have been shown to exhibit long-range interaction, leading to layering and jamming behavior at bottlenecks, reminiscent of Coulomb systems [5]. However, we are aware of no previous experimental studies of the transport of particles with strong Coulomb interactions though a controllable constriction, close to the limit of single particle transport. Here, we report transport measurements of strongly correlated electrons on the surface of liquid helium in which a split-gate electrode is employed to form a tunable constriction. Steplike increases in conductance are observed as the constriction is opened. We attribute each step to an increase in the number of electrons able to pass simultaneously though the constriction, due to the strong Coulomb interaction. Our results represent the classical counterpart of the transport of noninteracting Fermi gases in quantum point-contact devices [6].

Electrons are attracted to the surface of liquid helium by a small image charge but are prevented from entering the liquid by a potential barrier at the helium surface [7]. Motion perpendicular to the helium surface is quantized and at temperatures below $2 \mathrm{~K}$ electrons occupy the ground state, $11 \mathrm{~nm}$ above the liquid [8]. The in-plane mobility $\left(\sim 1 \times 10^{2} \mathrm{~m}^{2} / \mathrm{Vs}\right.$ at $\left.1 \mathrm{~K}\right)$ is determined by scattering with helium gas atoms and surface excitations (ripplons) [9]. For typical values of the surface density $n_{s}\left(10^{9}-10^{13} \mathrm{~m}^{-2}\right)$ the interelectron separation greatly exceeds the thermal electron wavelength at $1 \mathrm{~K}$. As the relative dielectric constant of the liquid helium is small $(\varepsilon=1.054)$, the Coulomb interaction between electrons is essentially unscreened. Thus, the system is nondegenerate and may be considered classical, the ground state being the 2D Wigner crystal [10].

Electrons on helium have been proposed as quantum bits with long coherence times [11]. More recently, superconducting microwave cavities have been proposed as readout devices for the electron lateral motional states and spin states [12]. Individual electrons in a microscopic trap have been counted using a single-electron transistor (SET) positioned beneath the helium surface [13]. Here, for the first time, we study the transport of single electrons on the surface of helium.

Two samples, samples 1 and 2, were studied in this experiment. Sample 1 is pictured in Fig. 1(a), and was fabricated on a silicon wafer using electron beam and optical lithography. Two arrays of 25 microchannels [14], each $20 \mu \mathrm{m}$ wide and connected in parallel, act as electron reservoirs. The two reservoirs are connected by a central channel of width $10 \mu \mathrm{m}$ and length $20 \mu \mathrm{m}$. The microchannels were filled by the capillary action of superfluid ${ }^{4}$ He. A $1.5-\mu \mathrm{m}$-thick layer of hard-baked photoresist defined the microchannel geometry and depth $d$. The surface of the photoresist was covered by the guard electrode. Electrodes covering the area beneath the helium in each reservoir were denoted as the left and right reservoir electrodes. A split-gate electrode, $1 \mu \mathrm{m}$ wide and separated by a gap of $2.8 \mu \mathrm{m}$, was positioned at the bottom of the central channel. The thin gap between left and right reservoir electrodes was placed $10 \mu \mathrm{m}$ to the right of the split gate in order not to distort the potential profile of the split-gate region. All the electrodes were of thickness $90 \mathrm{~nm}$ and made of gold. For sample 2 the split-gate width was $1.4 \mu \mathrm{m}$ and the separation was $2.6 \mu \mathrm{m}$. dc 

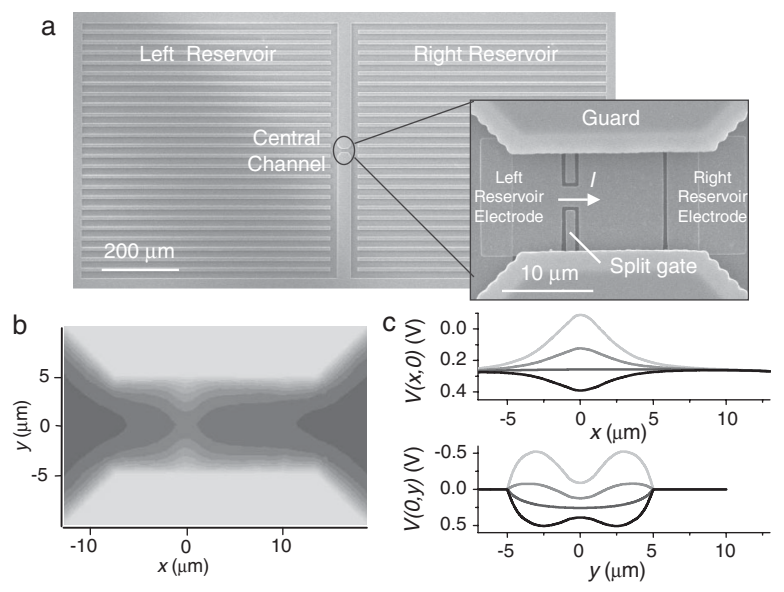

FIG. 1. (a) Scanning electron microscope image of sample 1. Inset: The split gate electrode in the central channel. (b) Contour plot of the electrostatic potential at the helium surface in the central channel calculated by FEM software for $V_{\mathrm{gu}}=V_{\mathrm{gt}}=$ $0 \mathrm{~V}, V_{r}=+0.3 \mathrm{~V}$. The darker colors indicate regions of more positive potential (lower energy for electrons). (c) Calculated potential along the channel $V(x, 0)$ and across the constriction $V(0, y)$ for $V_{\mathrm{gu}}=0 \mathrm{~V}, V_{r}=+0.3 \mathrm{~V}$ and $V_{\mathrm{gt}}=+1.3,+0.3$,

$0.7,2.3 \mathrm{~V}$ (dark to light grey). Note that the vertical axes are inverted.

bias voltages $V_{\mathrm{gu}}, V_{\mathrm{gt}}$ and $V_{r}$ were applied to the guard, split-gate, and reservoir electrodes, respectively. The helium surface was then charged with electrons by thermionic emission from a tungsten filament. The maximum surface electron density in the reservoirs was $n_{s}=$ $\varepsilon \varepsilon_{0}\left(V_{r}-V_{\mathrm{gu}}\right) / e d$, where $e$ is the elementary charge and $\varepsilon_{0}$ is the dielectric constant of vacuum.

A small ac voltage $V_{\text {in }}$ of frequency $200 \mathrm{kHz}$ was superimposed on the right reservoir electrode to drive electrons between the two reservoirs through the central channel. With reference to the standard lumped-circuit model [15], the peak-to-peak current $I$ and conductance $G$ of the electron system were measured by performing a phasesensitive measurement of the voltage capacitively induced on the left reservoir electrode. The temperature was $1.2 \mathrm{~K}$, at which the electron system remains in a liquidlike state.

We first describe a simple electrostatic model of the system. Note that in this model, a more positive voltage corresponds to a lower potential energy for an electron. Finite element modelling (FEM) software was used to simulate the electrostatic potential profile on the helium surface in the central channel. The results for the geometry of sample 1 are shown in Fig. 1(b). When $V_{\mathrm{gt}}$ is more negative than $V_{r}$, a saddle-point potential is formed between the split-gate electrodes, with a maximum in the $x$ direction along the channel and a minimum in the lateral $y$ direction [Fig. 1(c)], creating a constriction for electrons. We may write this potential as $V(x, y)=$ $V_{b}+\frac{1}{2} a x^{2}-\frac{1}{2} b y^{2}$, where $V_{b}$ is the potential at the center of the saddle point and $a$ and $b$ are constants. We will assume $a$ and $b$ depend on $V_{\mathrm{gu}}$ and $V_{r}$ but remain constant
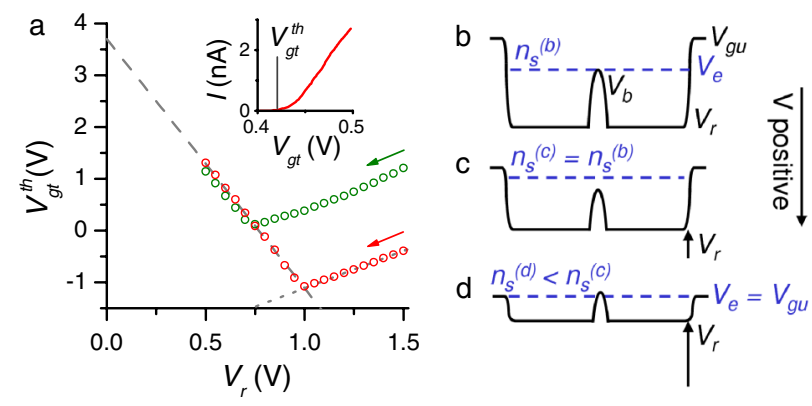

FIG. 2 (color online). Measurements with sample 1. (a) Measured values of $V_{\mathrm{gt}}^{\text {th }}$ with changing reservoir electrode voltage $V_{r}$ (positive to negative as indicated by the arrows) for $n_{s}=1.5 \times 10^{13} \mathrm{~m}^{2}$ (red circles) and $n_{s}=5.2 \times 10^{12} \mathrm{~m}^{2}$ (green circles). The dotted and dashed lines are linear fits to the data. Inset: the current $I$ decreases to zero at $V_{\mathrm{gt}}^{\mathrm{th}}$. (b) Schematic model of the electrostatic potential profile of the device at the current threshold $V_{e}=V_{b}$. (c) For $e V_{e}<e V_{\mathrm{gu}}$, the electron density remains constant as $V_{r}$ becomes more negative. (d) As electrons are lost to the guard, $V_{e}=V_{\mathrm{gu}}$.

over small variations in $V_{\mathrm{gt}}$. The reservoir, split-gate and guard electrodes have capacitances $C_{r}, C_{\mathrm{gt}}$, and $C_{\mathrm{gu}}$ respectively to the region of space at the center of the constriction $(x=0, y=0)$. The total capacitance of the region is then $C_{\Sigma}=C_{r}+C_{\mathrm{gt}}+C_{\mathrm{gu}}$ and we define constants to represent the relative strength of coupling from each electrode to the constriction region as $\alpha=C_{r} / C_{\Sigma}$, $\beta=C_{\mathrm{gt}} / C_{\Sigma}$ and $\gamma=C_{\mathrm{gu}} / C_{\Sigma}$. Then $V_{b}$ can be given by $V_{b}=\alpha V_{r}+\beta V_{\mathrm{gt}}+\gamma V_{\mathrm{gu}}$, where $\alpha+\beta+\gamma=1$. For sample 1(2) the FEM analysis gives the values $\alpha=$ $0.75(0.67), \beta=0.10(0.15), \gamma=0.15(0.18)$.

For electrons in the reservoirs, we assume that the electrostatic potential of the electron system $V_{e}$ depends on $V_{r}$ and on $n_{s}$ and can be written as $V_{e}=-e n_{s} d / \varepsilon \varepsilon_{0}+$ $V_{r}$. When $-e V_{e}>-e V_{b}$ electrons may pass through the constriction. However, as $V_{\mathrm{gt}}$ is swept negative $-e V_{b}$ increases and, when $-e V_{e}<-e V_{b}$, the electron transport is blocked by a potential barrier. Treating the electron system as a charge continuum, the threshold of current flow is therefore defined by the condition $V_{e}=V_{b}$. Such behavior has been demonstrated in preliminary measurements with a similar split-gate device [16].

Upon sweeping $V_{\mathrm{gt}}$ negative, the current flowing through the central channel decreased and then became zero at a threshold voltage $V_{\mathrm{gt}}^{\mathrm{th}}$, as expected from the electrostatic model when $V_{e}=V_{b}$. $V_{\mathrm{gt}}^{\mathrm{th}}$ was measured for different values of $V_{r}$ (from positive to negative) for $V_{\mathrm{gu}}=0 \mathrm{~V}$ and $V_{\text {in }}=$ $8 \mathrm{mV}_{\mathrm{pp}}$. The results of two such measurements, made with sample 1 after separate charging events, are shown in Fig. 2(a). In both cases, $V_{\mathrm{gt}}^{\text {th }}$ initially decreases as $V_{r}$ is decreased. Then, at a certain value of $V_{r}$, the trend is reversed as the threshold starts to increase. In Figs. 2(b)-2(d) we present a simple model to describe the dependence of $V_{\mathrm{gt}}^{\mathrm{th}}$ on $V_{r}$. We schematically show the electrostatic potential 
across the reservoirs through the central channel. In Fig. 2(b), at a positive reservoir bias and an appropriate split-gate bias so that $V_{e}=V_{b}$, the current is "pinchedoff." In Fig. 2(c), a decrease in $V_{r}$ changes both $V_{e}$ and $V_{b}$. The change in the barrier height $\Delta V_{b}\left(=\alpha \Delta V_{r}\right)$ is smaller than $\Delta V_{e}\left(=\Delta V_{r}\right)$, as $\alpha<1$. Therefore, $V_{\mathrm{gt}}$ must be set more negative to maintain the threshold condition. In Fig. 2(d), as $V_{r}$ decreases further, electrons are eventually lost to the guard electrode, leading to a reduction in $n_{s} . V_{e}$ now remains equal to the guard potential $V_{\mathrm{gu}}$. However, $V_{b}$ continues to change with $V_{r}$. $V_{\mathrm{gt}}$ must then be set more positive to maintain the threshold condition.

From this model and the threshold condition $V_{e}=V_{b}$, we may write down equations for the cases $-e V_{e}<$ $-e V_{\mathrm{gu}}$,

$$
V_{\mathrm{gt}}^{\mathrm{th}}=\frac{1-\alpha}{\beta} V_{r}-\frac{e n_{s} d / \varepsilon \varepsilon_{0}+\gamma V_{\mathrm{gu}}}{\beta},
$$

and $V_{e}=V_{\mathrm{gu}}$,

$$
V_{\mathrm{gt}}^{\mathrm{th}}=\frac{-\alpha}{\beta} V_{r}+\frac{1-\gamma}{\beta} V_{\mathrm{gu}} .
$$

For $-e V_{e}<-e V_{\mathrm{gu}}, V_{\mathrm{gt}}^{\mathrm{th}}$ depends on the electron density. In Fig. 2(a) the red circles represent measurements taken at the saturated density when $V_{r}=1 \mathrm{~V}$. The green circles represent a lower density, as $V_{\mathrm{gt}}^{\mathrm{th}}$ is more positive for $-e V_{e}<-e V_{\mathrm{gu}}$, before electrons are lost to the guard electrode at $V_{r}=0.7 \mathrm{~V}$. By making linear fits to the data, the coupling constants were determined as $\alpha=0.77$, $\beta=0.16, \gamma=0.07$, in good agreement with values given by the FEM analysis. The values measured for sample 2 were $\alpha=0.73, \beta=0.26, \gamma=0.01$, again in reasonable agreement with the FEM analysis and reflecting the influence of the sample geometry on the potential profile at the constriction. Changing $V_{\mathrm{gu}}, V_{\mathrm{gt}}$ and $V_{r}$ simultaneously by $+100 \mathrm{mV}$ caused a change in $V_{\mathrm{gt}}^{\mathrm{th}}$ of exactly $+100 \mathrm{mV}$, indicating that any additional stray capacitance to the barrier region was negligibly small.

In Fig. 2(a), upon extrapolation, $V_{\mathrm{gt}}^{\mathrm{th}}=3.6 \mathrm{~V}$ for $V_{r}=0 \mathrm{~V}$. We attribute this to an offset in $V_{\mathrm{gu}}$ of $V_{\mathrm{gu}}^{0} \approx$ $+0.62 \mathrm{~V}$, according to Eq. (2). Electrons were lost from the device for $V_{r}<0.5 \mathrm{~V}$, in approximate agreement with such an offset. A smaller offset of $\sim+0.10 \mathrm{~V}$ was observed for sample 2 . These offsets were found to be stable over many weeks and remained after thermally cycling the device to room temperature. In mesoscopic devices, potential offsets may be caused by contact or patch potentials, or by surface or image-charging effects [17]. For both samples we define the true value of the guard potential as $V_{\mathrm{gu}}^{\star}=V_{\mathrm{gu}}+V_{\mathrm{gu}}^{0}$. Using $V_{\mathrm{gu}}^{\star}$ in Eq. (2), the initial densities for the data shown in Fig. 2(a) are $n_{s}=1.5 \times 10^{13} \mathrm{~m}^{-2}$ (red circles) and $n_{s}=5.2 \times 10^{12} \mathrm{~m}^{-2}$ (green circles).

Figure 3(a) shows the conductance $G$ as $V_{\text {gt }}$ is swept for sample 1, for $V_{r}=1 \mathrm{~V}, V_{\mathrm{gu}}^{\star}=0.62 \mathrm{~V}$ and $V_{\text {in }}=4 \mathrm{mV}_{\mathrm{pp}}$. Weak steplike increases in $G$ are observed above the
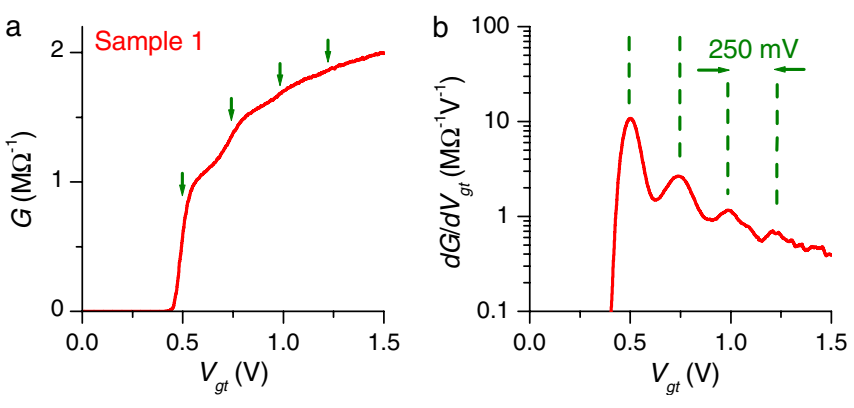

FIG. 3 (color online). Measurements with sample 1 for $V_{\mathrm{gu}}^{\star}=0.62 \mathrm{~V}, V_{r}=1 \mathrm{~V}$. (a) Conductance $G$ measured while sweeping $V_{\mathrm{gt}}$. The green arrows indicate the maxima in $d G / d V_{\mathrm{gt}}$. (b) Corresponding derivative $d G / d V_{\mathrm{gt}}$. The gradient at each point is calculated over a $50 \mathrm{mV}$ sampling window. The dashed lines indicate the peak spacing of $\sim 250 \mathrm{mV}$.

threshold. This structure can be seen more clearly in the derivative $d G / d V_{\mathrm{gt}}$ shown in Fig. 3(b). The spacing between steps is $\Delta V_{\mathrm{gt}} \approx 250 \mathrm{mV}$ which corresponds to $\Delta V_{b}=\beta \Delta V_{\mathrm{gt}} \approx 40 \mathrm{mV}$. In a quantum point contact, quantized increases in conductance appear as the Fermi energy of the electron system crosses each onedimensional subband. From the potential energy profile shown in Fig. 1(c) we estimate the energy of the subband spacing at the constriction to be $\sim 0.1 \mathrm{meV}$. Because the amplitude of $V_{\text {in }}$, and thus the fluctuation in $V_{e}$, greatly exceeds this energy scale, such features should be much too small to be resolved here.

We attribute the observed behavior to the ordering of electrons at the constriction due to Coulomb interaction. The constriction region may be modeled as a small square of width $w_{c}$ and area $S=w_{c}^{2}$. We assume that over this small area the potential is independent of $x(a=0)$, which is reasonable given that the FEM analysis indicates that $a \ll b$. The parabolic confinement is therefore given by $V(y)=V_{b}-\frac{1}{2} b y^{2}$. Again assuming a charge continuum, the electrons are distributed over the region for which $-e V(y)<-e V_{e}$. The constriction width is therefore defined as $w_{c}=\sqrt{8\left(V_{b}-V_{e}\right) / b}$. Using a parallel plate capacitor approximation, the charge density at the constriction may be written as $n_{c}=\varepsilon \varepsilon_{0}\left(V(y)-V_{e}\right) / e d$. Integrating this function over $x$ and $y$ between the limits $-w_{c} / 2$ and $+w_{c} / 2$ gives the total number of electrons in the constriction region as $N_{c}=16 \varepsilon \varepsilon_{0}\left(V_{b}-V_{e}\right)^{2} / 3 b e d$. The number of electrons across the constriction may then be estimated as $N_{y}=\sqrt{N} c$. From the FEM analysis we obtain $b=2.5 \times 10^{11} \mathrm{~V} / \mathrm{m}^{2}$, for $V_{r}-V_{\mathrm{gu}}^{\star}=0.38 \mathrm{~V}$. Using this value, and the relation $V_{b}-V_{e}=\beta\left(V_{\mathrm{gt}}-\right.$ $\left.V_{\mathrm{gt}}^{\mathrm{th}}\right)$, this analysis yields a linear increase in the number of electrons across the constriction as $V_{\mathrm{gt}}$ is swept positive, with a change in voltage of $\Delta V_{\mathrm{gt}}=225 \mathrm{mV}$ required to add each additional electron. We therefore conclude that the first step in the conductance corresponds to sequential single-electron transport through the constriction. We then 

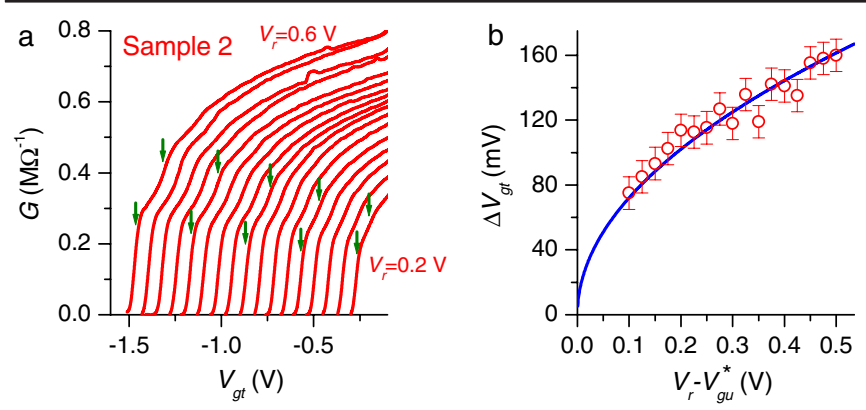

FIG. 4 (color online). Measurements with sample 2 for $V_{\mathrm{gu}}^{\star}=$ $0.1 \mathrm{~V}$. (a) Conductance $G$ with changing $V_{\mathrm{gt}}$, for $V_{r}=0.6$ to $0.2 \mathrm{~V}$ (left to right) in $25 \mathrm{mV}$ steps. The green arrows indicate the first and second maxima in $d G / d V_{\mathrm{gt}}$ for every fourth curve. (b) Measured separation between the first and second maxima in $d G / d V_{\mathrm{gt}}$ (red circles), and the calculated change in $V_{\mathrm{gt}}$ required to add an additional electron across the constriction (blue line), as a function of $V_{r} \quad V_{\mathrm{gu}}^{\star}$.

observe further steps in conductance when 2, 3 and finally 4 electrons are able to pass through the constriction simultaneously.

Figure 4(a) shows measurements taken with sample 2 for $V_{\text {in }}=4 \mathrm{mV}_{\mathrm{pp}}$. $G$ was measured as $V_{\mathrm{gt}}$ was swept, for $V_{\mathrm{gu}}^{\star}=0.1 \mathrm{~V}$ and different values of $V_{r} . V_{\mathrm{gt}}^{\text {th }}$ becomes more positive as $V_{r}$ decreases, indicating that electrons are lost to the guard and the electron density in the reservoirs decreases with each step in $V_{r}$. The steplike features observed in Fig. 3(a) are reproduced. The bias conditions for $V_{r}=$ $0.475 \mathrm{~V}\left(V_{r}-V_{\mathrm{gu}}^{\star}=0.375 \mathrm{~V}\right)$ and those used for sample 1 in Fig. 3(a) are similar. However, the spacing between the steps is smaller for sample $2\left(\Delta V_{\mathrm{gt}} \approx 140 \mathrm{mV}\right)$ and decreases further as $V_{r}$ decreases, as shown in Fig. 4(b). This is consistent with our model. Because $\beta=0.26$, larger than in sample 1, the electron density at the constriction changes more rapidly as $V_{\mathrm{gt}}$ is swept. Also, $b$ decreases as $V_{r}$ becomes less positive, because the parabolic confinement becomes flatter. The FEM analysis gives $b=6.84 \times 10^{11}\left(V_{r}-V_{\mathrm{gu}}^{\star}\right) \mathrm{V} / \mathrm{m}^{2}$. Using this expression, and the value $\beta=0.26, \Delta V_{\mathrm{gt}}$ was calculated in the same manner as for sample 1 , but as a function of $\left(V_{r}-V_{\mathrm{gu}}^{\star}\right)$. The result is in good agreement with the measured values of the step separation, as shown in Fig. 4(b).

The model used to calculate $\Delta V_{\mathrm{gt}}$, which takes into account the granularity of charge, implies that electron transport through the constriction should be blocked when $N_{y}<1$. The condition at the conduction threshold is then $V_{e}=V_{b}-\beta \Delta V_{\mathrm{gt}}$, rather than $V_{e}=V_{b}$ as was assumed in our initial charge continuum model. Taking this correction into account gives $\alpha=0.82(0.75), \beta=$ $0.15(0.24), \gamma=0.03(0.01)$ and $V_{\mathrm{gu}}^{0}=0.63(0.14)$ for sample 1(2). We find this correction to be relatively small, and also note that both the continuum model and the calculation of $\Delta V_{\text {gt }}$ make certain simplifying assumptions about the nature of transport through the constriction. Our results should be compared with those of numerical simulations of classical electron transport at a constriction to gain further insight into the electron dynamics close to the threshold.

In summary, we have measured the transport properties of a strongly correlated electron system on the surface of liquid helium at a point constriction formed by a split-gate electrode. Above the threshold of current flow, steplike increases in conductance are observed as the number of electrons that can pass simultaneously through the constriction increases. In this way, the electrons behave similarly to pedestrians at a bottleneck [18]. The stepwise increase in conductivity is similar to that observed in quantum point contacts, but in a quite different context. Our measurements allow a detailed characterisation of the saddle-point potential at the constriction and are in good agreement with a FEM analysis of the device.

We thank K. Ono, M. I. Dykman, F. Nori, and H. Totsuji for useful discussions. This work was partially supported by KAKENHI. DGR was supported by the RIKEN FPR Program.

\section{*drees@riken.jp}

${ }^{\dagger}$ CSNSM, Bâtiment 108, 91405 Orsay, France.

[1] N. Kokubo et al., Phys. Rev. Lett. 88, 247004 (2002).

[2] M. Köppl et al., Phys. Rev. Lett. 97, 208302 (2006).

[3] G. Piacente and F. M. Peeters, Phys. Rev. B 72, 205208 (2005).

[4] F. Bezanilla, Nat. Rev. Mol. Cell Biol. 9, 323 (2008).

[5] D. Helbing, I. Farkas, and T. Vicsek, Nature (London) 407, 487 (2000).

[6] C. W. J. Beenakker and H. van Houten, Solid State Phys. 44, 1 (1991).

[7] E. Andrei, Two Dimensional Electron Systems on Helium and Other Cryogenic Substrates (Kluwer Academic, Dordrecht, 1997).

[8] C. C. Grimes et al., Phys. Rev. B 13, 140 (1976).

[9] C. C. Grimes and G. Adams, Phys. Rev. Lett. 36, 145 (1976).

[10] C. C. Grimes and G. Adams, Phys. Rev. Lett. 42, 795 (1979).

[11] P. M. Platzman and M. I. Dykman, Science 284, 1967 (1999); S. A. Lyon, Phys. Rev. A 74, 052338 (2006).

[12] D. I. Schuster et al., Phys. Rev. Lett. 105, 040503 (2010).

[13] G. Papageorgiou et al., Appl. Phys. Lett. 86, 153106 (2005).

[14] P. Glasson et al., Phys. Rev. Lett. 87, 176802 (2001); H. Ikegami, H. Akimoto, and K. Kono, ibid. 102, 046807 (2009).

[15] Y. Iye, J. Low Temp. Phys. 40, 441 (1980).

[16] D. G. Rees and K. Kono, J. Low Temp. Phys. 158, 301 (2009).

[17] E. Rousseau et al., Phys. Rev. B 79, 045406 (2009).

[18] S. P. Hoogendoorn and W. Daamen, Transp. Sci. 39, 147 (2005). 